\title{
Immediate Extubation following Glenn Procedure for Single Ventricle Physiology: A Report of Three Cases
}

\author{
Grace G. Catalan
}

Department of Anesthesiology, College of Medicine and Philippine General Hospital, University of the Philippines Manila

\begin{abstract}
Postoperative mechanical ventilation after complex congenital cardiac surgery is still routine practice. Published reports on immediate extubation (in the operating room or $<6$ hours after surgery) appear safe and resulted in shorter intensive care unit and hospital stay. We present three cases of immediate extubation following a Glenn procedure in pediatric patients with single ventricle physiology. Our goal is to describe the successful surgical and anesthetic management and the potential benefits without increasing morbidity and mortality.
\end{abstract}

Key Words: congenital heart disease, Glenn procedure, pediatric, anesthesia, endotracheal extubation

\section{Introduction}

Immediate extubation (IE) following pediatric cardiac surgery is a strategy of removing the endotracheal tube after general anesthesia in the operating room or in the intensive care unit (ICU) within six hours post-operatively. Although often used in the context of fast track (FT) cardiac surgery, IE is not synonymous with, but is an integral component of fast tract cardiac surgery. FT is a protocol to reduce the perioperative course from hospital admission to discharge. It consists of admission on the morning of surgery, IE, brief ICU stay, and early hospital discharge. ${ }^{1}$

A systematic review and meta analysis of 23 studies on early extubation after pediatric cardiac surgery showed that IE was safe and was associated with the reduction in length of ICU and hospital stays without adverse effects on morbidity and mortality. The studies were conducted on neonates and infants up to five months on simple and complex cases such as atrial septal defect (ASD), ventricular septal defect (VSD), tetralogy of Fallot (TOF), and in patients with single ventricle physiology. The outcomes were the rate of reintubation, respiratory morbidity, early in-hospital mortality, and ICU and hospital length of stays and costs.

Corresponding author: Grace G. Catalan, MD

Department of Anesthesiology,

Philippine General Hospital

University of the Philippines Manila

Taft Avenue, Ermita, Manila 1000 Philippines

Telephone: +632 5548400 local 3320

Telefax: +6325242991

Email: gracegalila@yahoo.com
The predictors for failure of early extubation included younger age, longer cardiopulmonary bypass (CPB) time, and those needing circulatory arrest. ${ }^{2}$ Mittnacht et al., in a study on IE after congenital cardiac surgery on patients aged one month to eighteen years, identified two independent risk factors for failure to extubate in the operating room: younger age (<12 months) and a long CPB time $(>150$ minutes). ${ }^{3}$

To date, there are no recommended anesthetic techniques for IE after pediatric cardiac surgery. The data available are predominantly retrospective, observational and underpowered studies. There is also considerable variability in anesthesia techniques as well as heterogeneity in the subjects' age, physiology, and anatomic complexity. ${ }^{2}$

IE after pediatric cardiac surgery at the University of the Philippines, Philippine General Hospital (UP-PGH), is being practiced, albeit inconsistently, since 2004. This was prompted by the need for a more cost effective use of limited hospital and ICU resources and inspired by the successful experience in adult early extubation cardiac surgery practice that began in 2002.We started with atrial septal defects (ASD) and ventricular septal defects (VSD), followed by valvular defects and complex cyanotic cases, particularly tetralogy of Fallot (TOF). Lately, patients with single ventricle - tricuspid valve atresia (TVA), pulmonary valve atresia (PVA), hypoplastic right ventricle (RV), needing palliation were added. The most acceptable treatment is the Glenn procedure or the creation of a bidirectional cavopulmonary anastomosis to unload the pressure and volume in the functional ventricle. The goal of IE in these cases is to allow the patients to resume spontaneous ventilation to promote pulmonary blood flow, shorten recovery and limit ICU stay.

\section{Case Presentation}

We present three pediatric patients with a left ventricular form single ventricle physiology who underwent Glenn procedure under $\mathrm{CPB}$ using a general anesthesia technique followed by immediate extubation in the operating room. Data were taken from the electronic database of the department of surgery (ISIS) and anesthesia records of the section of thoracic and cardiovascular anesthesia of the UP-PGH. The same cardiac surgery and 
anesthesia team performed the procedures between June 2011 and April 2013.

The first patient was three years and five months old female, $11.8 \mathrm{~kg}$, diagnosed with tricuspid valve stenosis (TVS), PVA, hypoplastic RV. Balloon atrial septostomy (BAS) was done on the second day of life. The second patient was a three-year and nine-month-old male, $10.5 \mathrm{~kg}$, with a double outlet right ventricle (DORV), TVA, and pulmonary stenosis (PS). The third patient is a one year and sevenmonth-old female, $10.5 \mathrm{~kg}$, with severe TVS, hypoplastic RV, hypolastic PA. She underwent a modified Blalock-Taussig shunt (MBTS) at six months of age.

All patients were on regular follow-up and presented with poor weight gain, polycythemia, and cyanosis but with stable cardiopulmonary status preoperatively. The second patient had a seizure disorder controlled with oral phenobarbital while the other two patients did not have any comorbid conditions.

Anesthesia was induced using intravenous (IV) midazolam, ketamine, and fentanyl. Rocuronium was used to facilitate intubation in two patients and atracurium in one patient. Radial arterial catheters for continuous blood pressure monitoring and central venous catheters via the right internal jugular vein for central venous pressure monitoring were inserted. Anesthesia was maintained using sevoflurane in oxygen/air mixture and fentanyl (total dose: $10 \mu \mathrm{cg} / \mathrm{kg}$ ). Muscle relaxation was achieved by intermittent IV rocuronium or atracurium. After full heparinization, the aorta and vena cavae were cannulated, followed by CPB under moderate hypothermia. Continuous modified ultrafiltration (MUF) was initiated during bypass.

The surgery was done via a median sternotomy and was completed by dividing the superior vena cava from the atrium and anastomosing it to the pulmonary artery. Take down of the MBTS was done on the third patient. Total CPB times were 84 minutes, 85 minutes, and 114 minutes for the first, second and third patients respectively. Anesthesia was maintained during $\mathrm{CPB}$ by propofol infusion. Inotropic support, dobutamine or dopamine, was started during weaning from $\mathrm{CPB}$ and was maintained as required. Ventilation was resumed and oxygen saturation was maintained between 80 to $90 \%$. Blood and blood products were transfused as needed to correct coagulopathy and replace blood loss. At the conclusion of surgery, all patients received a dose of IV morphine and ketorolac or paracetamol for post-operative pain control. This was continued postoperatively. Neuromuscular blockade was reversed using IV atropine and neostigmine.

All patients were deemed ready for extubation after meeting the following criteria: bypass time of less than 150 minutes, hemodynamic stability with minimum inotropic support, normothermia, control of bleeding, good spontaneous respiratory effort with acceptable arterial blood gases (ABG), oxygen saturation above $80 \%$ in room air, awake and responsive, and with good pain control. The first patient was extubated fourteen minutes after the surgery and the second at five minutes after surgery, both in the operating room. The third patient was not extubated in the operating room although she met the criteria for extubation apart from the ongoing replacement of blood. She was safely extubated in the post-anesthesia care unit (PACU) eighty minutes post-surgery.

Close monitoring was continued overnight at the PACU. Oral feeding was resumed at two to four hours after extubation. The first two patients were sent back to regular rooms the morning after the surgery while the third was transferred to the pediatric ICU for additional monitoring. She was transferred to a regular room after 24 hours without any event. There were no reintubations, reoperations or any deterioration in hemodynamic and pulmonary function. They were discharged from the hospital on the fifth day post surgery. The total hospital stays were thirteen, eight, and seven days. A summary of the patient characteristics and their perioperative courses is presented in Tables 1 and 2.

Table 1. Patient Demographics

\begin{tabular}{|c|c|c|c|}
\hline & Case 1 & Case 2 & Case 3 \\
\hline $\begin{array}{l}\text { Age (years, } \\
\text { months) }\end{array}$ & 3,5 & 3,9 & 1,6 \\
\hline Sex & Female & Male & Female \\
\hline Weight (kg) & 11.8 & 10.5 & 7 \\
\hline $\begin{array}{l}\text { Cardiac } \\
\text { pathology }\end{array}$ & $\begin{array}{l}\text { TVS, PVA, } \\
\text { common atrium } \\
\text { hypoplastic RV, } \\
\text { S/P BAS }\end{array}$ & $\begin{array}{l}\text { DORV, PS, } \\
\text { TVA }\end{array}$ & $\begin{array}{l}\text { Severe TVS, } \\
\text { hypoplastic } \\
\text { RV, PA, S/P } \\
\text { BAS, MAPCAs }\end{array}$ \\
\hline Comorbidities & - & $\begin{array}{l}\text { Seizure } \\
\text { disorder }\end{array}$ & - \\
\hline
\end{tabular}

$\overline{\text { kg-kilograms; TVS-tricuspid valve stenosis; PVA-pulmonary valve atresia; } R V \text {-right }}$ ventricle; BAS-balloon atrial septostomy; DORV-double outlet right ventricle; PSpulmonary stenosis; TVA-tricuspid valve atresia; MAPCAs-major aortopulmonary collateral arteries

\section{Discussion}

The driving force behind the practice of IE after pediatric congenital cardiac surgery is largely health economics and the need for a more efficient utilization of ICU and hospital resources. ${ }^{1}$ Although IE in itself will not achieve these, a well-planned general anesthesia technique tailored towards immediate extubation will avoid the potential complications of mechanical ventilation and lessen the utilization of ICU resources and manpower and shorten ICU stay.

The variability in the anesthetic drugs and techniques in the published studies precludes recommending a standard anesthetic regimen for IE. Likewise, institutional practices and preferences influence choices. Techniques cited included the use of IV morphine, fentanyl, continuous propofol or morphine infusion, the use of long- acting muscle relaxant pancuronium, midazolam, and ketamine for induction. There were reports on the use of intrathecal and epidural morphine as adjunct to general anesthesia. In the cases we 
Table 2. Perioperative Course

\begin{tabular}{|c|c|c|c|}
\hline & Case 1 & Case 2 & Case 3 \\
\hline \multicolumn{4}{|l|}{ Anesthetic procedure } \\
\hline Premedication & - & - & - \\
\hline Induction & $\begin{array}{l}\text { Midazolam, ketamine, fentanyl, } \\
\text { Rocuronium }\end{array}$ & $\begin{array}{l}\text { Midazolam, ketamine, } \\
\text { fentanyl, Rocuronium }\end{array}$ & $\begin{array}{l}\text { Midazolam, ketamine, fentanyl, } \\
\text { atracurium }\end{array}$ \\
\hline Maintenance & $\begin{array}{l}\text { Sevo in O2/air, fentanyl, propofol, } \\
\text { rocuronium }\end{array}$ & $\begin{array}{l}\text { Sevo in O2/air, fentanyl, } \\
\text { propofol, Rocuronium }\end{array}$ & $\begin{array}{l}\text { Sevo in O2/air, fentanyl, propofol, } \\
\text { atracurium }\end{array}$ \\
\hline Conclusion & $\begin{array}{l}\text { Atropine, neostigmine, morphine, } \\
\text { ketorolac }\end{array}$ & $\begin{array}{l}\text { Atropine, neostigmine, } \\
\text { morphine, paracetamol }\end{array}$ & $\begin{array}{l}\text { Atropine, neostigmine, morphine, } \\
\text { ketorolac }\end{array}$ \\
\hline Post-op pain control & Nalbuphine, ketorolac & Morphine, paracetamol & Morphine, ketorolac \\
\hline Inotropic support & Dobutamine, NTG & Dopamine, NTG & Dobutamine, NTG \\
\hline Extubation time (minutes after surgery) & 14 & 5 & 80 \\
\hline Surgical procedure & $\begin{array}{l}\text { Atrial septectomy, ligation of main } \\
\text { pulmonary artery, Glenn shunt }\end{array}$ & Glenn shunt & $\begin{array}{l}\text { MBTS takedown, PDA ligation, } \\
\text { pulmonary arterioplasty, Glenn shunt }\end{array}$ \\
\hline CPB time (minutes) & 84 & 85 & 114 \\
\hline AX clamp time (minutes) & 8 & 69 & 81 \\
\hline Duration of surgery (hours, minutes) & 4,29 & 4,15 & 5,45 \\
\hline Intraoperative events & - & - & - \\
\hline Length of stay in CV PACU (hours) & 24 & 24 & 24 \\
\hline Length of stay in PICU (hours) & 0 (back to room) & 0 (back to room) & 24 \\
\hline Hospital LOS after surgery (days) & 5 & 5 & 6 \\
\hline Total hospital LOS (days) & 13 & 7 & 8 \\
\hline \multicolumn{4}{|l|}{ Outcomes } \\
\hline In-hospital mortality & - & - & - \\
\hline Need for reintubation & - & - & - \\
\hline Sepsis & - & - & - \\
\hline Reoperation & - & - & - \\
\hline Bleeding/coagulopathy & - & - & + \\
\hline Other events & - & - & - \\
\hline
\end{tabular}

presented, fentanyl and sevoflurane were used as the main anesthetic, and propofol infusion was used for maintenance of anesthesia during CPB. These drugs have favorable pharmacokinetics with early recovery, and did not produce significant respiratory and cardiac depression or excessive sedation at the conclusion of surgery. The muscle relaxants rocuronium and atracurium were routinely reversed with atropine and neostigmine. Morphine was used for analgesia because of its potent analgesic effect.

Improvement in surgical techniques, advancement in imaging, better understanding of the anatomy and pathophysiology all favor the IE strategy. Additionally, the use of moderate hypothermia, minimal priming volumes, modified and conventional ultrafiltration techniques of CPB reduce lung water and edema, and lessen inflammatory response facilitating early recovery from the surgery and anesthesia.

The decision for IE was based on inclusion and exclusion criteria extrapolated from the published reports. The criteria included preoperative patient characteristics, intraoperative events and hemodynamic stability and reversibility of anesthetics.

Extending the strategy of IE to patients with single ventricle specifically in TVA, PVA, and hypoplastic RV was based on the theoretical favorable pulmonary and hemodynamic effects of spontaneous breathing after the Glenn procedure. Anatomically, the left side of the heart is volume overloaded since it receives both the systemic and pulmonary blood flow. ${ }^{4}$ In the most common form, there is variable degree of obstruction to pulmonary blood flow and over time, the cyanosis worsens as the VSD closes or the infundibular obstruction increases. The goal is to optimize the physiologic state so these patients can undergo the Fontan procedure, which is the second and final surgery for a functional single ventricle. Symptomatic neonates require early palliation to create a systemic to pulmonary artery shunt, MBTS or if the ASD is restrictive, requires a BAS. This was done in two of the patients presented.

At six to eighteen months, a bidirectional cavopulmonary anastomosis or Glenn procedure is recommended. This will decrease the volume load on the heart and lower the pulmonary artery pressures making the patient a better candidate for the Fontan procedure. Because blood flow to the lungs becomes passive, resistance must be minimal for adequate flow to occur. Spontaneous ventilation will avoid increases in intrathoracic pressure thus promoting flow. Pulmonary vascular resistance should be kept low by optimizing functional residual capacity, increasing $\mathrm{FiO} 2$, and avoiding acidosis. ${ }^{5}$ 
Postoperatively, the decision to limit or bypass ICU stay was based on a stable and unsupported cardiopulmonary status. Those requiring respiratory assistance, inotropes, fluid boluses, or intensive monitoring and nursing care should stay in the ICU. After IE, there is greater patient and parent satisfaction, with earlier resumption of feeding and patient mobilization. ${ }^{3}$

Theoretically, we would expect a reduction in the overall hospital stay and cost. Although the time from surgery to hospital discharge was five days in all patients, the total hospital stay for the first patient was long (13 days) because of the time it took from admission to surgery. This underscores the need for a more efficient scheduling and pre-admission preparation of the patients. There were no available data on the hospitalization cost for the cases presented.

\section{Conclusion}

The practice of IE will simplify postoperative care, avoid complications of mechanical ventilation and increase patient and parent satisfaction. The more cost-effective use of ICU and hospital resources cannot be over emphasized. Current evidence shows that IE is safe and does not contribute to the morbidity and mortality in selected simple and complex cases. The benefits of IE following the Glenn procedure allows for early spontaneous ventilation promoting better blood flow to the pulmonary circulation.

We should look into the possibility of creating an immediate extubation protocol to include same day admission and short ICU stay in simple and complex pediatric congenital surgeries. We hope to validate this in a prospective study.

\section{References}

1. Howard F, Brown KL, Garside V, Walker I, Elliott MJ. Fast-track pediatric cardiac surgery: the feasibility and benefits of a protocol for uncomplicated cases. Eur J Cardiothorac Surg. 2010; 37(1):193-6.

2. Alghamdi AA, Singh SK, Hamilton BC, et al. Early extubation after pediatric cardiac surgery: systematic review, meta-analysis, and evidence-based recommendations. J Card Surg. 2010; 25(5):586-95.

3. Mittnacht AJ, Thanjan M, Srivastava S, et al. Extubation in the operating room after congenital heart surgery in children. J Thorac Cardiovasc Surg. 2008; 136(1):88-93.

4. Davies LK, Husain SA, Weitzel NS. Anesthetic management for patients with congenital heart disease: the pediatric population. In: Hensley FA, Martin DE, Gravlee GP, eds. Cardiac anesthesia, $5^{\text {th }}$ ed. Philadelphia: Lippincott Williams \& Wilkins; 2013. pp. 389-451.

5. Nicolson SC, Steven JM. Anesthesia for the patient with a single ventricle. In: Andropoulos DB, Stayer SA, Russell IA, eds. Anesthesia for Congenital Heart Disease. Malden: Blackwell Publishing, Inc.; 2005. pp. 356-372.

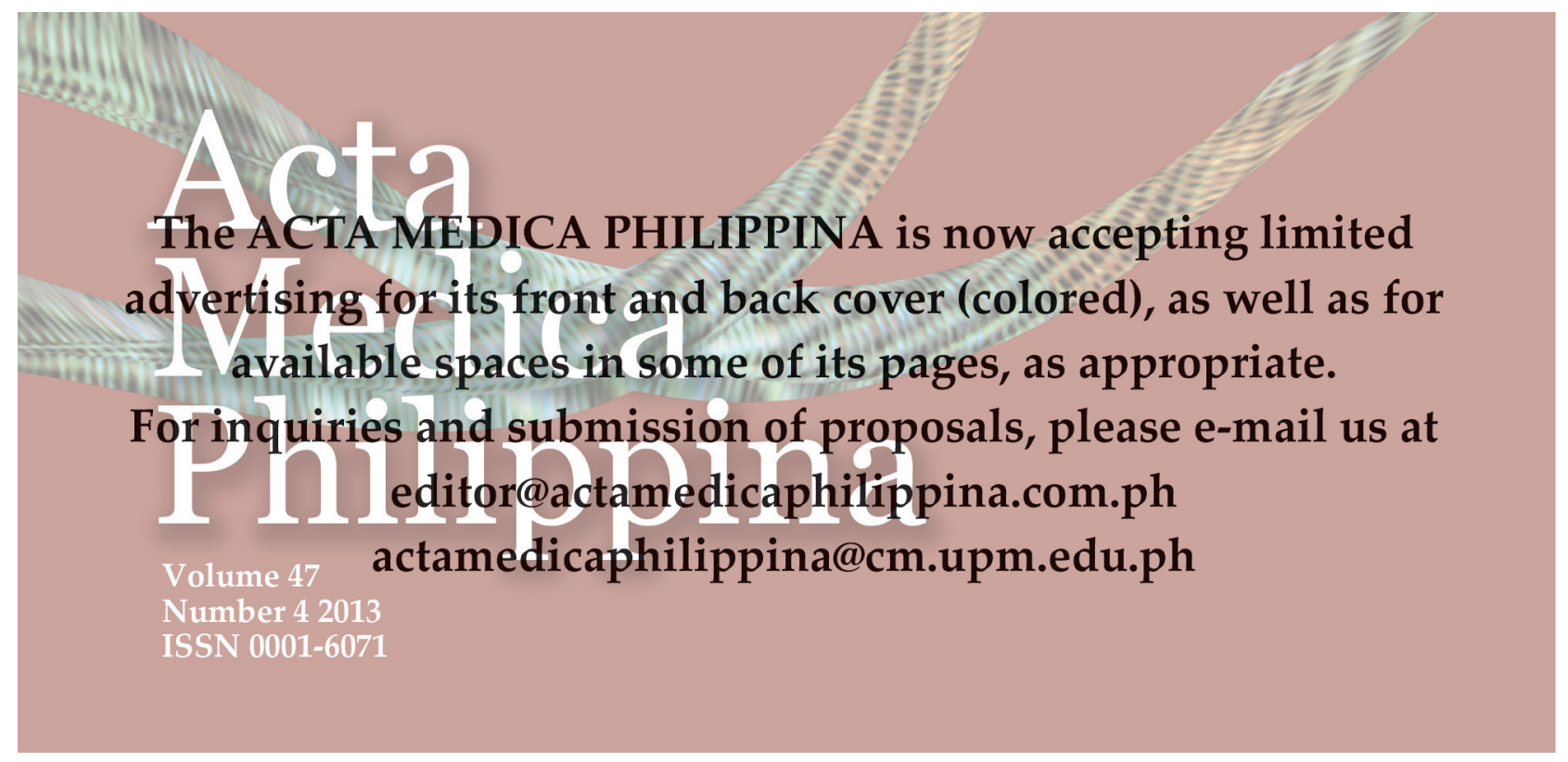

